diagnostic criteria selected were: Criterium 10 (ALT10) characterized by a RR $>23, \mathrm{HR}>85, \mathrm{~T}>37.5^{\circ} \mathrm{C}$ and Leukocytes $>10000$ or $<4000$ or bands $>5 \%$ or neutrophils $>0=70 \%$; Criterion 22 (ALT 22): RR $>23$, HR $>85, \mathrm{~T}$ $>37.8^{\circ} \mathrm{C}$, Leukocytes $>10000 \mathrm{o}<4000$ or bands $>5 \%$ or neutrophils $>0=$ $70 \%$. ALT10 and ALT 22 sensitivity were superior to that of classical criteria: $100 \%$ and $99.3 \%$ versus $95.1 \%$, respectively. Validation in hospitalized patients without sepsis revealed similar results, with ALT10 and ALT22 reaching 100\% sensitivity. Specificity remained around 83.7 to $84.8 \%$ when using the two alternative criteria in the derivation cohorts, while it was $72 \%$ when using classical criteria.

Conclusions.-Current sepsis criteria have limited specificity for diagnosis at high - altitude populations. The use of the new diagnostic criteria may decrease the rate of nondiagnosed patients from 5 to $0 \%$, while increasing specificity from 72 to $84.8 \%$.

\section{Cerebrovascular $\mathrm{CO}_{2}$ Reactivity: A Potential Tool for Prevention of High Altitude Brain Edema}

Jose Antonio Carmona Suazo

Hospital Juárez de México, México

In Rotterdam in 1996 with invasive neurological monitoring systems, such as brain tissue oxygen monitoring $\left(\mathrm{PbtiO}_{2}\right)$, jugular bulb oxygen catheters $\left(\mathrm{SavO}_{2} \mathrm{j}\right)$, intracranial pressure (ICP), exhaled $\mathrm{CO}_{2}$, and arterial blood gases in patients with severe cranial lesions, we designed a test to measure the $\mathrm{CO}_{2}$ reactivity. This test produced moderate hyperventilation, $\mathrm{CO}_{2}$ decreased from $35 \pm 2 \mathrm{~mm} \mathrm{Hg}$ to $31 \pm 3$ $\mathrm{mm} \mathrm{Hg}$. Since hyperventilation is a common therapeutic routine in severe head trauma, which decreases ICP, it is actually a controversial maneuver since that hyperventilation decreases $\mathrm{CBF}$ and oxygen delivery. $\mathrm{CO}_{2}$ reactivity is a physiological mechanism that allows for blood flow regulation in the brain according to metabolical requirements. For each $\mathrm{mmHg}$ of $\mathrm{CO}_{2}$ change, the blood flow is modified by $4 \mathrm{~mL}$. Hyperventilation results in vasoconstriction, while hypoventilation causes vasodilation. This auto regulation mechanism acts in the adaptation or failure to adapt to high altitude, and therefore can cause consciousness alterations when adaptation periods are too short. During hyperventilation at high altitude, $\mathrm{P}_{\mathrm{a}} \mathrm{CO}_{2}$ resulted in decreased brain oxygenation. It was more accentuated in later days than in the earlier ones. Near-infrared spectroscopy (NIRS) a noninvasive technique, measures the relationship between oxygen consumption and delivery from a mix of arterial and venous oxygen. The light passes through the skin, the skull, the cerebral parenchyma and makes it 4 to $5 \mathrm{~cm}$ into the dura mater with values of about 65 to $70 \%$ and the extreme values is less than $20 \%$. The Zubietas published tables to calculate changes in $\mathrm{CO}_{2}$ as environmental $\mathrm{O}_{2}$ decreases at increasing heights above sea level. These tables are quite useful; however, we do not have measurements determining the changes in brain blood flow inferred through studies of noninvasive multiparametric monitoring of oxygen, or other techniques such as bispectral index (BIS), let alone changes in blood flow following hyper- and hypoventilation at high altitude. We also lack data comparing healthy unadapted patients to native and adapted patients at different altitudes above sea level, nor do we have comparisons between adapted and unadapted patients at great heights exhibiting pathologies in which the relationship between supply and consumption of $\mathrm{O}_{2}$ is crucial. $\mathrm{CO}_{2}$ reactivity is the last mechanism lost in traumatic brain injury resulting in a dramatic outcome. Investigating this approach in noninvasive monitoring could be useful in HACE or HAPE cerebral edema and other pathologies presented by patients who either ascent to high altitude or descend from them abruptly, because $\mathrm{CO}_{2}$ reactivity could be used to prevent further damage.

Patients living at high altitudes have a $\mathrm{CO}_{2}$ autoregulation which is adapted to a certain level of $\mathrm{O}_{2}$ supply and consumption which therefore allows the patient to carry out multiple activities without manifesting clinical alterations. On the other hand, when unadapted individuals are abruptly exposed to high altitude, compensation between cerebral blood flow and $\mathrm{CO}_{2}$ levels is not regulated properly, which may result in abrupt changes in blood flow, which, depending on susceptibility, may in turn result in clinical manifestation that could be picked up by monitoring systems. Last year, in Cuzco (3200 m above sea level), we compared hyperventilation during 3 to 5 minutes between locals and unadapted foreigners. We measured exhaled $\mathrm{CO}_{2}$ levels up to $16 \mathrm{~mm} \mathrm{Hg}$ without inducing clinical or NIRS manifestations in those who were adapted to the height. In comparison, not being adapted to such heights, I presented signs of drowsiness and voice tone changes. It is with this antecedent that we have decided to present the same workshop in this symposium with different altitudes. We will have three objectives:
1. To demonstrate the reliability of our monitoring systems.

2. To describe the physiological changes measured with different monitoring systems-BIS, NIRS and exhaled $\mathrm{CO}_{2}$-and their correlations in locals and foreigners adapted to different altitudes.

3. To invite you to partake in a research project with this protocol, in which industries, universities or tourism could provide substantial support given the benefits they would obtain from the applications of these measurements.

\section{Vihangam Yoga Helps in High Altitude Chronic Hypoxia}

Nam Deo

Bangalore, India

The basic biochemical reaction for energy production in the body depends on oxygen. A minimum level of oxygen is essential at tissue level to sustain that basic metabolic reaction at the required rate. If oxygen falls below the critical level, the body system switches to an anaerobic process which cannot sustain life for long. This deficiency at tissue level is known as hypoxia. How can Vihangam yoga help in high altitude hypoxia? There are two options: either we maintain the supply or we decrease the oxygen demand: 1) Oxygen transport to supply at the required consumption; 2) Acclimatizing by increasing the hemoglobin concentration of the blood; 3) The way in which yoga can help is by reducing the demand by practicing yoga at level of life force (Prana), we can regulate our basic metabolic rate, hence we are reducing the demand. According to the Vihangam Yoga teachings, there are ten pranas (life forces) in our body, each one having its designated function as and when needed. Hence our basal metabolic rate (BMR) will be reduced, thus reducing the demand of oxygen needed to sustain life. It is not possible by other forms of yoga which only work at the level of the body. The age-old Vihangam yoga was rediscovered by HH Shri Sadguru Sadafaldeo ji and this has presently been propagated by HH Shri Sadguru Swatantradeo ji across the globe. It teaches us and trains us to use this method of meditation based on the intimate relationship between "Mind" and "Prana." By practicing it, our mind can be fully tamed and thereby, control over "Pranas" increases, which helps us in controlling and regulating our basal metabolic rate as discussed above, thus protecting us even from high altitude chronic hypoxia.

Media and Mountain Medicine: A Commitment: Role and Impact of Media on Society and with Special Reference to Newspapers' Role in Public Awareness

\section{Rajashekar Nanjappa}

\section{Regional General Manager, The Hindi Newspaper, Bengaluru, India}

Media is dynamic and plays an important and significant role in our society today. Media is all round us 24/7 and 365 days, in newspapers daily, weekly, or monthly; magazines, books, journals and periodicals; the television channels show news and programs on varied subjects be it education, agriculture, sports, science and technology, health and fitness, or entertainment. The radio broadcasts music, news, and public awareness programs on FM/MW/SW Radio stations. The new age of media includes the Internet and other social media: Twitter, Instagram, Facebook, and others. Media is a window to the world, without which people and the society would be ignorant and isolated from what is happening around them and in the world, including the highest human habitats like those in the cities of La Paz (3100 m to $4100 \mathrm{~m}$ ) and El Alto (4100 m). Media is present even on the summit of Mt Everest ( $8842 \mathrm{~m})$, the highest point in the world.

\section{Mountains-Law-Ethics}

\section{KVR Tagore}

\section{President LESAAT, Bengaluru, India}

"Don't look up at the mountain, climb it." Thus said Swami Vivekananda. Climb the mountain we can see the world, not so world can see us. When a goal matters enough to a person, that person will find a way to accomplish what first seemed impossible. Mountains therefore instigate us to make an adventurous attempt which has enormous environmental, socioeconomic and cultural value comprising vitally important resources and functions that benefit society both directly and indirectly: as regulators of climate and water resources; containing important water reserves and providing us with fuel as sanctuaries of a large diversities ecosystems, with unique and exclusive species and an extraordinary variety of landscapes, communities, languages, and cultures, as studying grounds for disciplines such as geology and biology and as ideal settings to develop 
environmental education activities. There has been a need to enact loss/ declarations on mountain protection having a large international tradition linked growing awareness of mountaineering community towards environment, as well as negative effects caused by the increase and mass marketing of mountains sports of tourism activities on mountain ecosystem and local communities. The article presents certain principles in believing that freedom to practice mountaineering depends on the enormous value of mountain areas which are often the sources of products essential to human kind as a whole. The role of mountain tourism is not only in supporting local communities but the misuse of existing resources may lead to the loss of biodiversity, massive or intrusive changes to the landscape, climate change and pollution due to overuse of sensitive areas. Several declarations made by various mountaineering organizations have insisted on accepting the risks and assure responsibility leading to the balance of goals with skills and equipment, adopting fair means and reporting honestly. The declarations also insist to strive for best practice and never stop learning by being tolerant, considerate and help each other for protecting the wild and natural character of mountains and cliffs in order to support local communities and their sustainable development. The values or otherwise termed as ethics of mountaineering connotes human dignity, life, liberty and happiness; intactness of nature, solidarity, self-actualization, truth and adventure. The attraction of sports climbing depends upon utilizing opportunities provided to improve climbing standards and techniques resulting in to experience the pure fun of climbing with the fear of falling greatly reduced and enhancing aspirations of climbers to take the first step on to rock outside in relative safety, gradually learning about widening their climbing experience and having broad based exposure to an adventurous elevated and higher vision improving the social behavior and to fight against the killers of the virgin nature it also improves to have better coexistence with the neighborhood in spite of diversities and multidirectional cultural variations The catastrophe resulted due to earthquake that shook Nepal living thousands of people standards in remote locations beyond the access of roads and helicopters; in a land of expedition the world food program brought food and relief items those in greatest needs opened an access to humanitarian relief. "Respect the mountains" is the key slogan to promote sustainable mountain tourism through a global network of volunteers who activated significant step in mountain protection in their respective territories. The risk, responsibility, duty of care, and liability insisted actions to reduce the risk of litigations against ourselves in taking actions to reduce the possibility of contributing any harms to others. Mountaineering enabled by legal and ethical foundation dictated that "If we were afraid of failures, we don't deserve success-we make a living by what we get, but we make a life but what we give." 